\title{
Polarization from magnetized optically thick accretion disks
}

\author{
N. A. Silant'ev ${ }^{1,2, \star}$ \\ 1 Instituto Nacional de Astrofísica, Óptica y Electrónica, Apartado Postal 51 y 216, Z.P. 72000, Pue. México \\ 2 Main Astronomical Observatory of Russian Academy of Sciences, Pulkovo, 196140 St.-Petersburg, Russia
}

Received 24 April 2001 / Accepted 25 October 2001

\begin{abstract}
We present simple approximate formulae for intensity and the other Stokes parameters of outgoing radiation for magnetized, plane-parallel optically thick atmospheres. We assume that the polarization arises as a result of the light scattering on free electrons in magnetized plasma of an atmosphere. Our asymptotic formulae take place when the Faraday rotation angle $\psi$ at the optical length $\tau \approx 1$ is large, $\psi \geq 1$, and magnetic field $\leq 10^{5} \mathrm{G}$, when the scattering cross-section has the Thomson value. The formulae describe the radiation for an arbitrary direction of the magnetic field. The Milne problem, the atmospheres with homogeneous and linearly distributed thermal sources, as well as exponential sources are considered. The superposition of these "standard" sources can represent practically any real distribution of sources in optically thick accretion disks. Polarization for some particular models of magnetic fields on the surface of flat accretion disks are considered (chaotic fields in turbulent plasma, axially symmetric plane magnetic fields and the field of the magnetic dipole). The presented results allow us to calculate or estimate the polarization for a large variety of optically thick magnetized accretion disks.
\end{abstract}

Key words. accretion disks - polarization - magnetic fields - stars - quasars - galaxies: nuclei

\section{Introduction}

The existence of accretion disks around very different objects - neutron stars, white dwarfs, young stars, close double systems, quasars and active galactic nuclei, is a commonly accepted paradigm (see Frank et al. 1995). Due to the absence of axial symmetry with respect to our line of sight, the total integrated radiation coming out of accretion disks is polarized. There are numerous observations of light polarization from all the objects mentioned above (see, for example, Stockman et al. 1984; More \& Stockman 1984; Bastien \& Ménard 1988, 1990; Webb et al. 1993; Korotkar et al. 1995; Yudin 1996; Schmidt et al. 1997; Hutsemékers et al. 1998). The existence of magnetic fields in various cosmic objects is also shown (see the vast review of Vallée 1997). No doubt, many accretion disks are magnetized. Nevertheless, it is difficult to show the existence of a magnetic field in any particular source. Our simple formulae give rise to some characteristic dependence of the polarization spectra on the wave-length and can be used to search for magnetic fields in accretion disks.

The theory of radiative transfer in magnetized atmospheres is developed in a number of papers (Basko 1977; Silant'ev 1979, 1982, 1994; Meszaros et al. 1980; Kaminker et al. 1982). For the case of large magnetic fields, $B>$ $10^{5} \div 10^{6} \mathrm{G}$, when the cross-sections of optical radiation

\footnotetext{
* e-mail: silant@inaoep.mx
}

differ from the Thomson value $\sigma_{\mathrm{Th}} \simeq 6.65 \times 10^{-25} \mathrm{~cm}^{2}$, one can neglect the Faraday rotation of elliptically polarized normal modes and consider the system of coupled equations for the intensities of these modes (Nagel 1980; Kaminker et al. 1982). The different intensities of these normal waves give rise to the net polarization of the radiation. For weaker magnetic fields, $B<10^{5} \mathrm{G}$, the normal waves are right and left circular polarized and have Thomson scattering cross-sections. The anisotropy of the magnetized atmosphere in this case is due to the Faraday rotation only. Such atmospheres were considered in the papers (Silant'ev 1994; Agol \& Blaes 1996; Agol et al. 1998). In this paper we consider only this case.

In Silant'ev (1994) the principle of invariance (Chandrasekhar 1950) and the Sobolev technique (Sobolev 1960) were used to obtain the exact solutions of the problems: the Milne problem (the sources are far below the boundary), atmospheres with the power, $\sim \tau^{n}$, and exponential, $\sim \exp (-h \tau)$, sources. Here, $\tau$ is the total optical length, including the scattering and absorption processes. The solutions are expressed in terms of tensor $H$-functions, which satisfy the nonlinear matrix integral equation. For an oblique magnetic field this equation is very complex.

In Agol \& Blaes (1996) and Agol et al. (1998) the Milne problem and the atmosphere with linearly distributed, $\sim(1+\beta \tau)$, thermal sources were considered numerically 
(Monte Carlo method, the Feautrier technique). The magnetic field was directed along the normal to an atmosphere. We note the very interesting discussion on the influence of the photon true absorbtion in processes of polarization and depolarization (Agol et al. 1998).

The angle of the Faraday rotation $\psi$ may be written in the form (Gnedin \& Silant'ev 1997):

$\psi(\boldsymbol{n}, \boldsymbol{B})=\frac{1}{2} \delta \tau_{\mathrm{Th}} \cos \Theta \equiv \frac{1}{2} \delta(1-q) \tau \cos \Theta$,

$\delta=\frac{3 \lambda}{4 \pi r_{\mathrm{e}}} \cdot \frac{\omega_{B}}{\omega} \simeq 0.8 \lambda^{2}(\mu \mathrm{m}) B(G)$,

where $\boldsymbol{n}$ is the direction of wave propagation, $\Theta$ is the angle between $\boldsymbol{n}$ and magnetic field $\boldsymbol{B}, \tau_{\mathrm{Th}}=N_{\mathrm{e}} \sigma_{\mathrm{Th}} l$ is the Thomson optical length, $\sigma_{\mathrm{Th}}=8 \pi r_{\mathrm{e}}^{2} / 3$ is the Thomson cross-section, $r_{\mathrm{e}}=e^{2} / m c^{2} \approx 2.82 \times 10^{-13} \mathrm{~cm}$ is classic radius of electron, $N_{\mathrm{e}}$ is the electron number density, $l$ is the geometric length, $\omega_{B}=e B / m c$ is the cyclotron frequency, $\omega=2 \pi c / \lambda=k c$ is cyclic frequency of radiation $\left(\omega_{B} / \omega \cong 0.93 \times 10^{-8} \lambda(\mu \mathrm{m}) B(G)\right), q$ is the degree of true absorption.

Photons escape the optically thick atmosphere mainly from the boundary layer with $\tau \approx 1$. If the Faraday rotation angle $\psi$ corresponding to this optical length is about unity, then the outgoing radiation will be depolarized as a result of summation of radiation fluxes with very different angles of Faraday's rotation. Only for directions of the light propagation with $\Theta \approx 90^{\circ}$ the Faraday rotation angle is small, or absent, and depolarization does not occur. The diffusion of light in the inner parts of an atmosphere depolarizes the radiation even in the absence of a magnetic field, as a result of multiple photon scattering. For this reason, far from the boundary, radiation is unpolarized. The existence of the Faraday rotation only increases the depolarization process. Thus, the polarization of outgoing radiation acquires the peak-like angular dependence with its maximum for $\boldsymbol{n} \perp \boldsymbol{B}$. The sharpness of the peak increases with the increasing of magnetic field values and is of the order $\Delta \theta \approx 1 /((1-q) \delta)$.

As is known, the polarization terms (the Stokes parameters $Q$ and $U$ ) weakly influence the intensity of radiation $I$. So, the solution of the transfer equation for the intensity $I$ only (with the Rayleigh phase function) gives $I\left(\vartheta=0^{\circ}\right) / I\left(\vartheta=90^{\circ}\right)=3.02$ for the Milne problem with $q=0$. The same problem accounting for the polarization terms gives the value 3.06 . Here, $\vartheta$ is the angle between the outward normal $\boldsymbol{N}$ to the surface of the atmosphere and the direction $\boldsymbol{n}$ of the light propagation $(\cos \vartheta=\mu)$. Furthermore, the peak-like polarization terms for the case of a magnetized atmosphere are practically insignificant when determining the intensity $I$. This means that the intensity of radiation $I(\tau, \boldsymbol{n})$ in the atmospheres with sufficient Faraday's rotation $((1-q) \delta \geq 1)$ can be calculated from the separate transfer equation with the Rayleigh phase function. Thus, for such atmospheres, the intensity $I(\tau, \boldsymbol{n})$ does not depend on the magnetic field value.
The contribution of the polarization terms $Q$ and $U$ by calculation of the polarization itself is rather large for atmospheres without a magnetic field. So, the calculation of the polarization using the known intensity of radiation for the Milne problem gives the value $9.37 \%$, instead of the known value of $11.71 \%$. It means that the difference, $11.71 \%-9.37 \%=2.34 \%$ ( $20 \%$ of total polarization), is due to the contribution of the polarization terms in the transfer equation. In the presence of the Faraday rotation, contribution of the polarization terms is much smaller and tends to zero with the increase of the parameter $(1-q) \delta$. This contribution can be estimated as $\sim 20 \% /(1+(1-q) \delta)$. Thus, for $(1-q) \delta \geq 1$ we can neglect this contribution and calculate the linear polarization using the known intensity of radiation. Such an approach gives us the opportunity to easily calculate the polarization of light for an arbitrary direction of magnetic field and gives rise to simple analytical formulae.

The aim of this paper is to give simple formulae for parameters $Q$ and $U$ for an arbitrary magnetic field inclination. The only restriction is $(1-q) \delta \geq 1$. We give the solutions of the following problems: the Milne problem in the presence of absorbtion $(q \neq 0)$; a planeparallel atmosphere with the sources $S(\tau)$ of thermal radiation of the forms: $S(\tau)=q S_{0}, S(\tau)=q S_{1} \tau$, and $S(\tau)=q S_{h} \exp (-h \tau)(h=0.5,1,2,5)$. The linear superposition of these standard sources can approximate practically all forms of real sources in accretion disks.

We use the general theory (Silant'ev 1994) which acquires a very simple form for the large Faraday rotation case, considered in this paper. We present here only the final simplified formulae of this theory. The advantage of our approach, using the principle of invariance and Sobolev's technique, is that we can obtain the azimuthal dependence of polarization for an oblique magnetic field in an analytical form. In this aproach it is not required to solve the radiative transfer equation for particular values of the azimuthal angle. Finally, the observed intensity and polarization of outgoing radiation are expressed in terms of one scalar $H$-function, which is tabulated for a number of values of the absorption degree $q$. The exactness of our formulae can be estimated by the comparison with the results of the numerical calculations of Agol et al. (1998) for the Milne problem. The comparison shows that when $(1-q) \delta \geq 50$ our asymptotic formulae coincide with the exact numerical calculations. For $(1-q) \delta \approx 10$ they are accurate to $\approx 10 \%$. For the limiting case $\delta=0$ (atmosphere without magnetic field) our asymptotic formulae only qualitatively correspond to the exact values of polarization presented in the papers Silant'ev (1980) and Loskutov \& Sobolev (1981). So, for $q=0$ first, at $\mu \approx 0$, asymptotic value is less by $20 \%$ the real polarization, and then, when $\mu \longrightarrow 1$ and polarization tends to zero, our asymptotic polarization is twice the exact value. For $q=0.1-0.5$ the asymptotic values are less to $\approx 40 \%$ than exact polarization. 


\section{The basic formulae}

As was discussed, the intensity $I(\tau, \mu)$ in the case of large Faraday rotation obeys the separate transfer equation with Rayleigh's phase function $(3 / 16 \pi)(1-q)\left(1+\left(\boldsymbol{n} \cdot \boldsymbol{n}^{\prime}\right)^{2}\right)$. We consider only the case of thermal sources when this equation acquires the form:

$$
\begin{aligned}
& \frac{\mathrm{d} I(\tau, \mu)}{\mathrm{d} \tau}=I(\tau, \mu) \\
& -\frac{3}{16}(1-q) \int_{-1}^{1} \mathrm{~d} \mu^{\prime}\left(3-\mu^{2}-\mu^{\prime 2}+3 \mu^{2} \mu^{\prime 2}\right) I\left(\tau, \mu^{\prime}\right)-S(\tau) .
\end{aligned}
$$

Here, $\mu=\cos \vartheta, \mu^{\prime}=\cos \vartheta^{\prime}$ with $\vartheta$ and $\vartheta^{\prime}$ being the angles between the outward normal $\boldsymbol{N}$ to the plane-parallel atmosphere and directions of propagation of incident $\boldsymbol{n}$ and scattered $\boldsymbol{n}^{\prime}$ waves.

The principle of invariance (Chandrasekhar 1950), applied to Eq. (2), gives rise to the system of coupled nonlinear equations for two $H$-functions, $A(\mu)$ and $B(\mu)$. Sobolev (1972) showed that these functions can be expressed in terms of one $H$-function. This function satisfies the usual nonlinear equation for $H$-functions:

$H(\mu)=1+\mu H(\mu) \int_{0}^{1} \mathrm{~d} \mu^{\prime} \Psi\left(\mu^{\prime}\right) H\left(\mu^{\prime}\right) /\left(\mu+\mu^{\prime}\right)$

with $\Psi(\mu)$ being equal to:

$\Psi(\mu)=\frac{3}{16}(1-q)\left[3-(1+q) \mu^{2}+3 q \mu^{4}\right]$.

The explicit formulae for $A(\mu)$ and $B(\mu)$ (Silant'ev 1994) are:

$$
\begin{aligned}
& A(\mu)=\left[1+\mu^{2}+(3 / 2)(1-q) \mu\left(B_{1}-\mu B_{0}\right)\right] H(\mu) / 4, \\
& B(\mu)=\left[1-\mu^{2}-(3 / 2)(1-q) \mu\left(A_{1}-\mu A_{0}\right)\right] H(\mu) / 2,
\end{aligned}
$$

where

$$
\begin{aligned}
& A_{n}=\int_{0}^{1} \mathrm{~d} x x^{n} A(x), \quad B_{n}=\int_{0}^{1} \mathrm{~d} x x^{n} B(x), \\
& H_{n}=\int_{0}^{1} \mathrm{~d} x x^{n} H(x) .
\end{aligned}
$$

The moments $A_{n}$ and $B_{n}$ are connected with $H_{n}$ :

$$
\begin{aligned}
A_{0}= & {\left[2\left(H_{0}+H_{2}\right)\right.} \\
& \left.+(3 / 2)(1-q)\left(H_{1}^{2}+H_{2}^{2}-H_{1} H_{3}-H_{0} H_{2}\right)\right] / \Delta, \\
B_{0}= & {\left[4\left(H_{0}-H_{2}\right)\right.} \\
& \left.+(3 / 2)(1-q)\left(H_{2}^{2}-H_{1}^{2}+H_{0} H_{2}-H_{1} H_{3}\right)\right] / \Delta, \\
A_{1}= & {\left[2\left(H_{1}+H_{3}\right)+(3 / 2)(1-q)\left(H_{1} H_{2}-H_{0} H_{3}\right)\right] / \Delta, } \\
B_{1}= & {\left[4\left(H_{1}-H_{3}\right)+(3 / 2)(1-q)\left(H_{0} H_{3}-H_{1} H_{2}\right)\right] / \Delta, } \\
\Delta= & 8+9(1-q)^{2}\left(H_{2}^{2}-H_{1} H_{3}\right) / 4 .
\end{aligned}
$$

Using the very effective technique of Chandrasekhar (1950), we calculated all the functions for a number of values $q$. The values $A(\mu)$ and $B(\mu)$ are presented in Table 1. For $\mu=0 A(0)=0.25$ and $B(0)=0.5$. If $q \longrightarrow 1$, the $A(\mu)$ - function tends to $\left(1+\mu^{2}\right) / 4$ and $B(\mu) \longrightarrow\left(1-\mu^{2}\right) / 2$. Now we can give the explicit formulae for the intensity and polarization for various problems. The values of the Stokes parameters $Q$ and $U$ depend on the choice of the reference system. In this paper we use the system with the $x$-axis lying in the plane $(\boldsymbol{n} \boldsymbol{N})$. So, a negative $Q$ corresponds to the electric field oscillations being perpendicular to this plane.

\subsection{The Milne problem}

The Milne problem deals with the case when sources are in very deep layers of an atmosphere $(\tau \gg 1)$. According to the general theory (Silant'ev 1994) we have for intensity $I(\mu)\left(\mathrm{erg} \mathrm{cm}^{-2} \mathrm{~s}^{-1} \mathrm{~Hz}^{-1}\right.$ sterad $\left.^{-1}\right)$ and the Stokes parameters $Q(\boldsymbol{n}, \boldsymbol{B})$ and $U(\boldsymbol{n}, \boldsymbol{B})$ the following expressions:

$$
\begin{aligned}
& I^{(\mathrm{M})}(\mu)=\frac{F}{2 \pi J_{1}} J(\mu), \\
& J(\mu)=\frac{2}{1+g} \cdot \frac{2 A(\mu)+g B(\mu)}{1-k \mu}, \\
& Q^{(\mathrm{M})}(\boldsymbol{n}, \boldsymbol{B})= \\
& \quad-\frac{F}{2 \pi J_{1}} \cdot \frac{1-g}{1+g} \cdot \frac{\left(1-\mu^{2}\right)(1-k \mu)}{(1-k \mu)^{2}+(1-q)^{2} \delta^{2} \cos ^{2} \Theta},
\end{aligned}
$$

$$
\begin{aligned}
& U^{(\mathrm{M})}(\boldsymbol{n}, \boldsymbol{B})= \\
& \quad-\frac{F}{2 \pi J_{1}} \cdot \frac{1-g}{1+g} \cdot \frac{\left(1-\mu^{2}\right)(1-q) \delta \cos \Theta}{(1-k \mu)^{2}+(1-q)^{2} \delta^{2} \cos ^{2} \Theta} .
\end{aligned}
$$

Here, $F$ is the flux of radiation, $J_{1}$ is the first moment of the function $J(\mu)$ (see Eq. (6) as a definition of the moments). The $J(\mu)$ - function describes the angular distribution of outgoing radiation $(J(\mu) \equiv I(\mu) / I(0))$. The parametrs $g$ and $k$ are determined from the solution of a homogeneous system of two equations (see Silant'ev 1994). The characteristic value $k=k(q)$ is the root of the characteristic equation of this system. The values of $J(\mu), g$, $k$ and $J_{1}$, are presented in Table 2. For small $q$ one has $k \simeq \sqrt{3 q}$ and $k \longrightarrow 1$ for $q \longrightarrow 1$. The characteristic feature of the angular distribution of outgoing radiation is the extended form along the normal $\boldsymbol{N}$. The sharpness of this distribution increases with an increase of the degree of absorption $q$. Mathematically this feature is described by the denominator $(1-k \mu)$ in $(8)$. This behaviour is a consequence of the fact that the density of radiation grows as $\exp (k \tau)$ for sufficiently large optical depth. Note that for the conservative photon scattering $(q=0)$ the radiation inside an atmosphere propagates in accordance with the diffusion law, $\sim \tau$. The greater absorption creates the sharper distribution of the radiation density near the boundary, and, as a consequence, the sharpness of the angular distribution of outgoing radiation increases. 
Table 1. The values of the functions $A(\mu)$ and $B(\mu)$.

\begin{tabular}{|c|c|c|c|c|c|c|c|c|c|c|c|c|c|c|}
\hline \multirow[b]{2}{*}{$\mu$} & \multicolumn{2}{|c|}{$q=0$} & \multicolumn{2}{|c|}{0.05} & \multicolumn{2}{|c|}{0.1} & \multicolumn{2}{|c|}{0.2} & \multicolumn{2}{|c|}{0.3} & \multicolumn{2}{|c|}{0.4} & \multicolumn{2}{|c|}{0.5} \\
\hline & $A$ & $B$ & $A$ & $B$ & $A$ & $B$ & $A$ & $B$ & $A$ & $B$ & $A$ & $B$ & $A$ & $B$ \\
\hline 0 & 5 & & 55 & & 25 & 0 & 25 & 0.5 & 25 & U & 25 & & .25 & 0.5 \\
\hline 0.05 & 2940 & 9 & 2856 & & 817 & 0 & 2760 & 4 & 2715 & 0 & 0.2676 & & 0.2642 & 5190 \\
\hline 0.10 & 3322 & 5938 & 146 & 65 & 3069 & 0.5678 & 0.2961 & 46 & 2878 & & .2809 & & 0.2749 & 268 \\
\hline 0.15 & 3698 & & 21 & & & & & & & & 34 & & 852 & \\
\hline 0.20 & 0.4076 & 6499 & 3689 & $c_{1}$ & & 0.59 & & 5730 & 3178 & & 3057 & & 0.2956 & 0.5273 \\
\hline 0.25 & 0.4459 & 0.6709 & 56 & 6238 & 63 & 0.60 & & 49 & & & & & 0.3064 & 0.5217 \\
\hline 0.30 & 0.4850 & 0.6879 & 4223 & 0.6299 & 0.3990 & 0.60 & 0.3689 & 0.5 & 78 & & 14 & 00 & 79 & 126 \\
\hline 0.3 & 0.5249 & & 93 & & 0 & 0.6 & & & & & & & 01 & 04 \\
\hline 0.40 & 657 & & 66 & 0 & & & & & & & & & & 851 \\
\hline 0.45 & 0.6075 & 07180 & 5043 & 0.6243 & & & & & & & & & & 0.4667 \\
\hline 0.50 & 0.6503 & 0.7214 & 0.5325 & 0.6151 & 0.4933 & 0.57 & 0.4457 & 0.5 & 0.4143 & 0.4929 & 0.3908 & 0.4 & 0.3721 & 0.4455 \\
\hline 0.55 & 0.6942 & 0.7217 & .5612 & 0.6024 & 0.5181 & 0.5 & 0.4665 & 70 & 0.4328 & 0.4 & 0.4078 & & 0.3 & 0.4213 \\
\hline 0.60 & 0.7391 & 39 & 5905 & 2 & 36 & 0 . & 80 & 39 & 22 & 61 & 58 & & 50 & 0.3944 \\
\hline 0.65 & 0.7851 & 0.7131 & 0.6205 & 0.5667 & 0.5698 & 0.5176 & 0.5104 & 0.4577 & 0.4725 & & 0.4448 & & 0.4230 & 0.3646 \\
\hline 0.70 & 0.8322 & 0.7043 & 0.6512 & 0.5438 & 0.5967 & 0.4915 & 0.5336 & 0.4284 & 0.4937 & 0.3872 & 0.4648 & 0.3565 & 0.4421 & 0.3321 \\
\hline 0.75 & & & & & & & & & & & & & 0.4623 & 0.2968 \\
\hline 0.80 & 0.9297 & 0.6778 & 0.7146 & 0.4884 & 0.6527 & 0.4297 & 0.5826 & 0.3608 & 0.5392 & 0.3167 & 0.5079 & 0.2843 & 0.4836 & 0.2588 \\
\hline 0.85 & 0.9800 & 0.6602 & 0.7475 & 0.4559 & 0.6820 & 0.3942 & 0.6086 & 0.3225 & 0.5634 & 0.2771 & 0.5311 & 0.2440 & 0.5061 & 0.2181 \\
\hline 0.90 & 1.0316 & 0.6396 & 0.7812 & 0.4203 & 0.7121 & 0.3556 & 0.6355 & 0.2813 & 0.5887 & 0.2347 & 0.5554 & 0.2010 & 0.5297 & 0.1747 \\
\hline 0.9 & 1.0842 & 0.6162 & & 0.3815 & 0.7432 & 0.31 & & & & & & & & 0.1286 \\
\hline 1 & 1.1380 & 0.5898 & 0.8509 & 0.3396 & 0.7751 & 0.2692 & 0.6923 & 0.1901 & 0.6425 & 0.1415 & 0.6074 & 0.1067 & 0.5804 & 0.0799 \\
\hline
\end{tabular}
form:

The degree of polarization $p=\left(Q^{2}+U^{2}\right)^{1 / 2} / I$ has the

$$
\begin{aligned}
& p^{(\mathrm{M})}(\boldsymbol{n}, \boldsymbol{B})= \\
& \quad \frac{1-g}{1+g} \cdot \frac{1-\mu^{2}}{J(\mu)} \cdot \frac{1}{\sqrt{(1-k \mu)^{2}+(1-q)^{2} \delta^{2} \cos ^{2} \Theta}} .
\end{aligned}
$$

The position angle $\chi$ of the polarization plane is determined from the relation:

$\tan 2 \chi=\frac{U}{Q}=\frac{(1-q) \delta}{1-k \mu} \cdot \cos \Theta$.

In accordance with the discussion in the Introduction, the maximum degree of polarization $p_{\max }^{(\mathrm{M})}(\mu)$ occurs at $\boldsymbol{n} \perp \boldsymbol{B}$ $(\cos \Theta=0)$, where the Faraday rotation is absent. This polarization, as a function of $\mu$, is presented in Fig. 1. For $q=0,0.5$ and $\boldsymbol{B} \| \boldsymbol{N}$ we demonstrate also how the Faraday rotation transforms the polarization curves into peaked curves. These curves correspond to $(1-q) \delta=10$. For greater values of this parameter the sharpness of $p(\mu)$ increases. For an oblique magnetic field the condition $\boldsymbol{n} \perp$ $\boldsymbol{B}$ occurs at $\mu \neq 0$. Our curves allow us to establish the maximum polarization for these cases. Note that for $q \longrightarrow$ 1 the coefficient $g \longrightarrow 0$ and $p_{\max }^{(\mathrm{M})}(0) \longrightarrow 100 \%$.

For maximum polarization $(\cos \Theta=0)$ one has $Q^{(\mathrm{M})}<$ 0 and $U^{(\mathrm{M})}=0$, i.e. the electric field oscillations are perpendicular to the plane $(\boldsymbol{n} \boldsymbol{N})$. Because the degree of absorption $q=q(\lambda)$ depends on the wavelength, the known dependence $U / Q \sim \lambda^{2}$ exists only in nonabsorbing atmospheres with $q=0$ (for more detailed discussion, see Agol et al. 1998).



Fig. 1. Polarization for the Milne problem. Solid lines present maximum polarization $p_{\max }^{(\mathrm{M})}(\mu)$ for $\boldsymbol{n} \perp \boldsymbol{B}$. The numbers denote the values of the absorption degree $q$. Dotted lines demonstrate the peak-like polarization for $(1-q) \delta=10$ and $\boldsymbol{B} \| \boldsymbol{N}$. The dashed line presents Chandrasekhar's polarization for a non-magnetized and non-absorbing atmosphere.

\subsection{Homogeneous sources}

If the sources of radiation are distributed homogeneously, $S(\tau)=q S_{0}$, the formulae for the intensity and polarization of outgoing radiation acquire the form:

$I^{(0)}(\mu)=q S_{0}\left[2 \alpha_{0} A(\mu)+\beta_{0} B(\mu)\right]$ 
Table 2. The values of the function $J(\mu)$ and the coefficients $k, g$ and $J_{1}$ for the Milne problem.

\begin{tabular}{|c|c|c|c|c|c|c|c|}
\hline \multirow[b]{2}{*}{$\mu$} & \multirow{2}{*}{$\begin{array}{l}q=0 \\
J\end{array}$} & \multirow{2}{*}{$\begin{array}{l}0.05 \\
J\end{array}$} & \multirow{2}{*}{$\begin{array}{l}0.1 \\
J\end{array}$} & \multirow{2}{*}{$\begin{array}{l}0.2 \\
J\end{array}$} & \multirow{2}{*}{$\begin{array}{ll} & 0.3 \\
J & \\
\end{array}$} & \multirow{2}{*}{$\begin{array}{l}0.4 \\
J\end{array}$} & \multirow{2}{*}{$\begin{array}{l}0.5 \\
J\end{array}$} \\
\hline & & & & & & & \\
\hline 0 & 1 & 1 & 1 & 1 & 1 & 1 & 1 \\
\hline 0.05 & 1.1467 & 1.1427 & 1.1386 & 1.1301 & 1.1213 & 1.1122 & 1.1030 \\
\hline 0.10 & 1.2647 & 1.2591 & 1.2532 & 1.2407 & 1.2274 & 1.2133 & 1.1986 \\
\hline 0.15 & 1.3746 & 1.3691 & 1.3631 & 1.3496 & 1.3342 & 1.3173 & 1.2992 \\
\hline 0.20 & 1.4801 & 1.4766 & 1.4721 & 1.4606 & 1.4459 & 1.4284 & 1.4087 \\
\hline 0.25 & 1.5829 & 1.5832 & 1.5822 & 1.5761 & 1.5651 & 1.5495 & 1.5303 \\
\hline 0.30 & 1.6836 & 1.6901 & 1.6946 & 1.6981 & 1.6942 & 1.6836 & 1.6674 \\
\hline 0.35 & 1.7829 & 1.7979 & 1.8105 & 1.8282 & 1.8359 & 1.8340 & 1.8239 \\
\hline 0.40 & 1.8811 & 1.9074 & 1.9308 & 1.9684 & 1.9930 & 2.0047 & 2.0048 \\
\hline 0.45 & 1.9784 & 2.0190 & 2.0565 & 2.1208 & 2.1692 & 2.2009 & 2.2166 \\
\hline 0.50 & 2.0750 & 2.1332 & 2.1884 & 2.2878 & 2.3689 & 2.4290 & 2.4680 \\
\hline 0.55 & 2.1710 & 2.2504 & 2.3277 & 2.4723 & 2.5976 & 2.6981 & 2.7709 \\
\hline 0.60 & 2.2666 & 2.3711 & 2.4754 & 2.6778 & 2.8629 & 3.0202 & 3.1428 \\
\hline 0.65 & 2.3617 & 2.4958 & 2.6328 & 2.9088 & 3.1745 & 3.4128 & 3.6092 \\
\hline 0.70 & 2.4564 & 2.6250 & 2.8014 & 3.1709 & 3.5462 & 3.9017 & 4.2104 \\
\hline 0.75 & 2.5509 & 2.7592 & 2.9828 & 3.4712 & 3.9974 & 4.5267 & 5.0128 \\
\hline 0.80 & 2.6451 & 2.8989 & 3.1789 & 3.8193 & 4.5569 & 5.3529 & 6.1348 \\
\hline 0.85 & 2.7390 & 3.0447 & 3.3919 & 4.2281 & 5.2688 & 6.4945 & 7.8096 \\
\hline 0.90 & 2.8328 & 3.1972 & 3.6246 & 4.7151 & 6.2049 & 8.1718 & 10.5709 \\
\hline 0.95 & 2.9264 & 3.3573 & 3.8802 & 5.3059 & 7.4904 & 10.8723 & 15.9632 \\
\hline 1 & 3.0198 & 3.5257 & 4.1626 & 6.0376 & 9.3641 & 15.9318 & 31.0925 \\
\hline$k$ & 0 & 0.378659 & 0.523200 & 0.704828 & 0.819984 & 0.896901 & 0.947380 \\
\hline$g$ & 0.83255 & 0.80264 & 0.77129 & 0.70405 & 0.63055 & 0.55063 & 0.46437 \\
\hline$J_{1}$ & 1.19402 & 1.29277 & 1.40518 & 1.68238 & 2.05696 & 2.58190 & 3.35377 \\
\hline
\end{tabular}

$$
Q^{(0)}(\boldsymbol{n}, \boldsymbol{B})=-q S_{0} \frac{1-\mu^{2}}{2} \cdot \frac{\alpha_{0}-\beta_{0}}{1+(1-q)^{2} \delta^{2} \cos ^{2} \Theta},
$$

$$
U^{(0)}(\boldsymbol{n}, \boldsymbol{B})=-q S_{0} \frac{1-\mu^{2}}{2} \cdot \frac{\left(\alpha_{0}-\beta_{0}\right)(1-q) \delta \cos \Theta}{1+(1-q)^{2} \delta^{2} \cos ^{2} \Theta}
$$

The coefficients $\alpha_{0}$ and $\beta_{0}$ are presented in Table 3. For $q \longrightarrow 1$ these coefficients tend to unity. This means that $I^{(0)} \longrightarrow q S_{0}\left(J^{(0)}(\mu) \longrightarrow 1\right)$ and the polarization tends to zero. At the opposite limit $q \longrightarrow 0$ the angular distribution $J^{(0))}(\mu) \equiv I^{(0)}(\mu) / I^{(0)}(0)$ and the degree of polarization coincides with those of the Milne problem. This is quite natural because the main contribution to the intensity of outgoing radiation is from the distant parts of an atmosphere and the situation looks like the Milne problem. This is true for all distributions of thermal sources which increase inside an atmosphere, particularly for linearly distributed sources (see next subsection). For $q \longrightarrow 1$ the contribution of scattered radiation (which is partly polarized) tends to zero and the polarization of outgoing radiation also disappears. Figure 2 illustrates this clearly. This figure also presents the maximum polarization $p_{\max }^{(0)}(\mu)$ corresponding to $\cos \Theta=0$. For $q=0.01$ and $\boldsymbol{B} \| \boldsymbol{N}$ we give the transformed curves of the polarization for $(1-q) \delta=10$ and 50 . It is seen that the increase of the magnetic field (remember that $\delta \sim B$ ) gives rise to a sharper form of the polarization curve.

\subsection{The case of linearly distributed sources}

Now we give the formulae for sources of the form $S(\tau)=$ $q S_{1} \tau$ :

$$
\begin{aligned}
& I^{(1)}(\mu)=q S_{1}\left[2\left(\alpha_{1}+\mu \alpha_{0}\right) A(\mu)+\left(\beta_{1}+\mu \beta_{0}\right) B(\mu)\right] \\
& Q^{(1)}(\boldsymbol{n}, \boldsymbol{B})= \\
& -q S_{1} \frac{1-\mu^{2}}{2} \cdot\left[\frac{\left(\alpha_{0}-\beta_{0}\right) \mu\left(1-(1-q)^{2} \delta^{2} \cos ^{2} \Theta\right)}{\left(1+(1-q)^{2} \delta^{2} \cos ^{2} \Theta\right)^{2}}\right. \\
& \left.\quad+\frac{\alpha_{1}-\beta_{1}}{1+(1-q)^{2} \delta^{2} \cos ^{2} \Theta}\right] \\
& U^{(1)}(\boldsymbol{n}, \boldsymbol{B})=-q S_{1} \frac{1-\mu^{2}}{2} \cdot\left[\frac{2 \mu\left(\alpha_{0}-\beta_{0}\right)}{\left(1+(1-q)^{2} \delta^{2} \cos ^{2} \Theta\right)^{2}}\right. \\
& \left.\quad+\frac{\alpha_{1}-\beta_{1}}{1+(1-q)^{2} \delta^{2} \cos ^{2} \Theta}\right](1-q) \delta \cos \Theta
\end{aligned}
$$

The coefficients $\alpha_{1}$ and $\beta_{1}$ are also presented in Table 3 (the third and fourth lines from the top). For $q \longrightarrow 0$ the fraction $\beta_{1} / \alpha_{1} \longrightarrow 0.83255$, which is the $g$ - coefficient for the Milne problem (the same is true for $\beta_{0} / \alpha_{0}$ in the previous case). Thus, the angular distribution $J^{(1)}(\mu)$ and the polarization $p_{\max }^{(1)}(\mu)$ tend to those of the Milne problem. Because linearly growing thermal sources are closer to the latter, this coincidence of the angular distribution and polarization occurs up to larger values of $q(q \approx 0.1)$ than for homogeneous sources. 
Table 3. The values of the coefficients $\alpha$ and $\beta$ for various problems. The first two upper lines correspond to the case of homogeneous sources. The next two lines describe the linearly distributed sources, and the others refer to the exponential sources.

\begin{tabular}{lllllllllllll}
\hline$q$ & 0.01 & 0.05 & 0.1 & 0.2 & 0.3 & 0.4 & 0.5 & 0.6 & 0.7 & 0.8 & 0.9 & 0.99 \\
\hline$\alpha_{0}$ & 10.41642 & 4.59871 & 3.22726 & 2.26350 & 1.83976 & 1.58872 & 1.41832 & 1.29312 & 1.19622 & 1.11841 & 1.05417 & 1.00504 \\
$\beta_{0}$ & 9.11007 & 4.20755 & 3.02812 & 2.18017 & 1.79738 & 1.56586 & 1.40596 & 1.28673 & 1.19323 & 1.11728 & 1.05393 & 1.00504 \\
\hline$\alpha_{1}$ & 53.71482 & 9.08645 & 3.95636 & 1.58043 & 0.85654 & 0.52000 & 0.33067 & 0.21171 & 0.13137 & 0.07425 & 0.03207 & 0.00285 \\
$\beta_{1}$ & 44.55025 & 7.43478 & 3.18844 & 1.23946 & 0.65542 & 0.38894 & 0.24208 & 0.15188 & 0.09243 & 0.05128 & 0.02176 & 0.00190 \\
\hline$\alpha_{0.5}$ & 3.51342 & 2.62789 & 2.20445 & 1.79188 & 1.56578 & 1.41507 & 1.30452 & 1.21860 & 1.14916 & 1.09142 & 1.04238 & 1.00398 \\
$\beta_{0.5}$ & 3.34751 & 2.57104 & 2.18634 & 1.79904 & 1.57984 & 1.43029 & 1.31855 & 1.23037 & 1.15818 & 1.09747 & 1.04539 & 1.00428 \\
$\alpha_{1}$ & 2.48587 & 2.07557 & 1.84303 & 1.58814 & 1.43472 & 1.32641 & 1.24366 & 1.17731 & 1.12234 & 1.07569 & 1.03537 & 1.00334 \\
$\beta_{1}$ & 2.46209 & 2.09256 & 1.87347 & 1.62382 & 1.46810 & 1.35539 & 1.26762 & 1.19615 & 1.13616 & 1.08468 & 1.03974 & 1.00377 \\
$\alpha_{2}$ & 1.83887 & 1.65720 & 1.54014 & 1.39788 & 1.30425 & 1.23413 & 1.17815 & 1.13167 & 1.09204 & 1.05758 & 1.02716 & 1.00259 \\
$\beta_{2}$ & 1.88210 & 1.71258 & 1.59693 & 1.44977 & 1.34897 & 1.27144 & 1.20828 & 1.15501 & 1.10899 & 1.06853 & 1.03247 & 1.00310 \\
$\alpha_{5}$ & 1.38258 & 1.31945 & 1.27401 & 1.21302 & 1.16898 & 1.13375 & 1.10412 & 1.07845 & 1.05576 & 1.03540 & 1.01692 & 1.00163 \\
$\beta_{5}$ & 1.44277 & 1.38058 & 1.33241 & 1.26403 & 1.21237 & 1.16982 & 1.13328 & 1.10110 & 1.07226 & 1.04610 & 1.02213 & 1.00214 \\
\hline
\end{tabular}

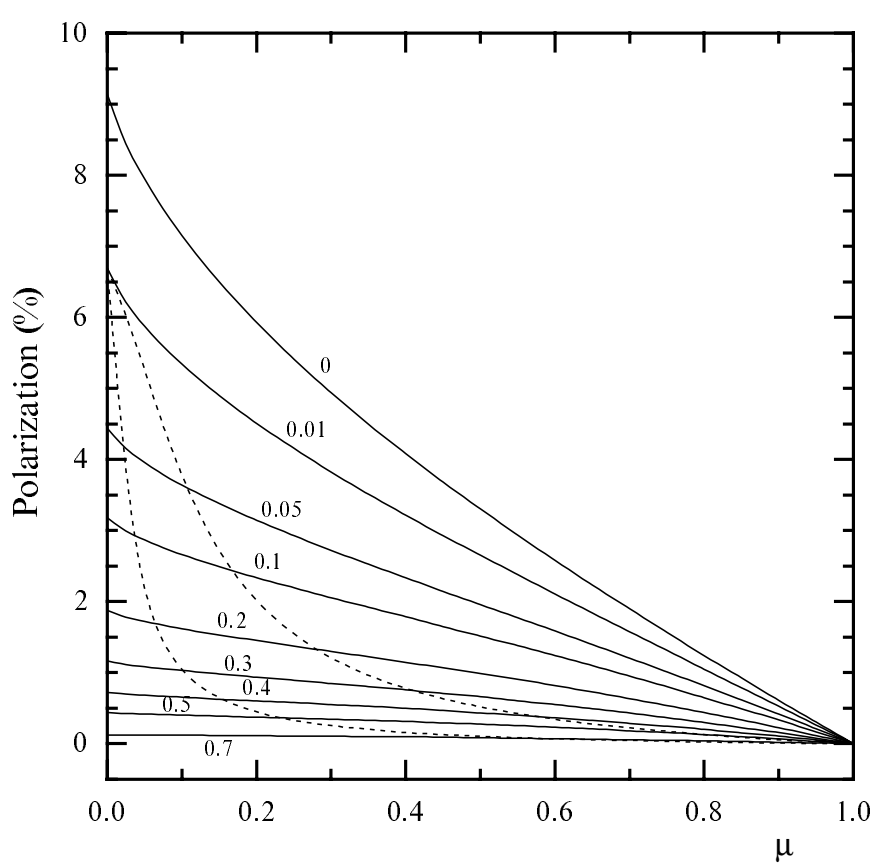

Fig. 2. Polarization for an atmosphere with homogeneous sources. The notations are the same as in Fig. 1. The solid lines present maximum polarization $p_{\max }^{(0)}(\mu)$ for $\boldsymbol{n} \perp \boldsymbol{B}$. The upper and low dotted lines present the polarization for $(1-q) \delta=10$ and 50, respectively.

In contrast to homogeneous sources, the angular distribution $J^{(1)}(\mu)$ acquires a sharper form with the increase of the absorption coefficient $q$, as happens for the Milne problem. For $q \longrightarrow 1$ the coefficients $\alpha_{1}$ and $\beta_{1}$ tend to zero such that $\beta_{1} / \alpha_{1} \longrightarrow 3 / 2$. This means that $p_{\max }^{(1)}(0)$ tends to $20 \%$, in contrast to the Milne problem, where this polarization tends to $100 \%$. Another difference with the Milne problem is the peak-like form of $p_{\max }^{(1)}(\mu)$ for $q \longrightarrow 1$. Note that for the Milne problem both the intensity $I^{(\mathrm{M})}(\mu)$ and the Stokes parameters $Q^{(\mathrm{M})}(\mu)$ and $U^{(\mathrm{M})}(\mu)$ include the characteristic denominator $(1-k \mu)$, i.e. they, even for

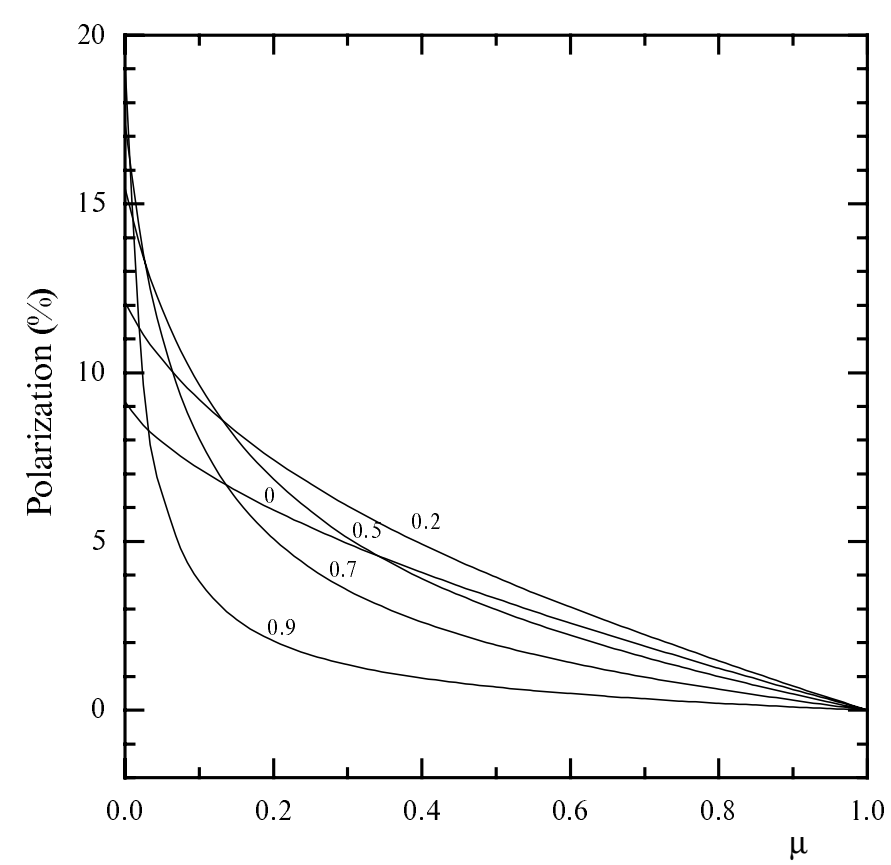

Fig. 3. Polarization for an atmosphere with linearly distributed sources. The notations are the same as in Fig. 1. The solid lines present maximum polarization $p_{\max }^{(1)}(\mu)$ for $\boldsymbol{n} \perp \boldsymbol{B}$.

$\cos \Theta=0$, are very sharp functions of $\mu$ for $q \longrightarrow 1$. But the polarization degree $p^{(\mathrm{M})}(\boldsymbol{n} ; \boldsymbol{B})$ does not depend on this value and is proportional to $\left(1-\mu^{2}\right)$ for large values of $q$. On the contrary, the $Q^{(1)}$ and $U^{(1)}$ at $\cos \Theta=0$ are not sharp compared with $I^{(1)}$. This explains the sharpness of the angular distribution of the maximum polarization $p_{\max }^{(1)}(\mu)$. These characteristic features are seen in Fig. 3. Of course, the Faraday rotation additionally increases the peak-like form of the polarization curves. 


\subsection{The exponential sources}

These sources can arise if the surface of an atmosphere is bombarded by fluxes of particles and radiation. Also, they can be used to approximate the real distribution of sources in an atmosphere. For such sources, $S(\tau)=q S_{h} \exp (-h \tau)$, we give:

$$
\begin{aligned}
& I^{(h)}(\mu)=q S_{h}\left[2 \alpha_{h} A(\mu)+\beta_{h} B(\mu)\right] /(1+h \mu), \\
& Q^{(h)}(\boldsymbol{n}, \boldsymbol{B})= \\
& \quad-q S_{h} \frac{1-\mu^{2}}{2} \cdot \frac{\left(\alpha_{h}-\beta_{h}\right)(1+h \mu)}{(1+h \mu)^{2}+(1-q)^{2} \delta^{2} \cos ^{2} \Theta}, \\
& U^{(h)}(\boldsymbol{n}, \boldsymbol{B})= \\
& \quad-q S_{h} \frac{1-\mu^{2}}{2} \cdot \frac{\left(\alpha_{h}-\beta_{h}\right)(1-q) \delta \cos \Theta}{(1+h \mu)^{2}+(1-q)^{2} \delta^{2} \cos ^{2} \Theta} .
\end{aligned}
$$

The coefficients $\alpha_{h}$ and $\beta_{h}$ for $h=0.5,1,2$ and 5 are given in Table 3 (below the first four lines). First, we note that these sources exist near the atmosphere's surface and the diffusion regime of the photon propagation does not occur even for the case of a non-absorbing medium. Direct escape of radiation from the atmosphere here plays a more important role than in the cases considered above. Because the thermal sources give unpolarized radiation, the outgoing radiation will be less polarized than in the previous three cases. Roughly speaking, we may imagine these sources as distributed in boundary layers near the surface of an atmosphere.

If the thickness of such layer is small (the cases $h=2$ and 5), the angular distribution of outgoing radiation is extended for directions near the surface, compared to the direction along the normal $\boldsymbol{N}$. For the first directions we collect the photons from the line of sight, where the sources exist ad infinitum. On the other hand, for the direction along the normal, the density of radiation decreases rapidly beyond the optical depth $\sim 1 / h$.

For thicker layers ( $h=0.5$ and 1$)$ the situation is more complex. First, for small absorption the angular distribution is extended along the normal $\boldsymbol{N}$. So, we have $J^{(1 / 2)}(1)=1.955$ and $J^{(1)}(1)=1.446$ for $q=0$. Then, with the increase of $q$, the values $J(1)$ diminish and the maximum of $J(\mu)$ tends to $\mu \approx 0.5(q \approx 0.2$ for $h=0.5$ and $q \approx 0.05$ for $h=1)$. After that $(q \approx 0.3$ and $q \approx 0.1$, respectively) the $J(1)$ - values became less than unity. For large $q(q \approx 0.7$ and $q \approx 0.4)$ these angular distributions monotonically decrease from the standard value 1 at $\mu=0$.

It is natural that polarization depends on the particular form of the angular distribution of photons near the surface. Figures 4 and 5 reflect this dependence. As usual, we present the maximum degree of polarization $p_{\max }^{(h)}(\mu)$, corresponding to $\cos \Theta=0$.

First, consider Fig. 4 for the case $h=0.5$. Because for small $q$ the angular distribution of photons is extended along the normal $\boldsymbol{N}$, the scattered radiation is polarized

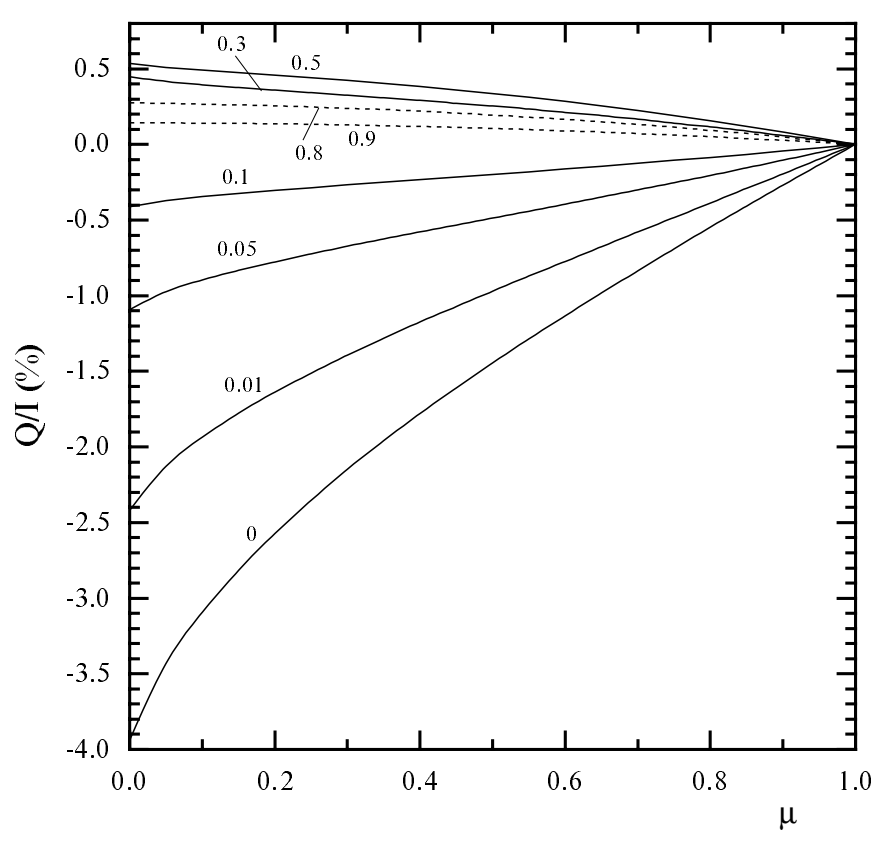

Fig. 4. The Stokes parameter $Q_{\max }^{(1 / 2)}(\mu)$ for an atmosphere with exponential sources $(h=0.5)$ and for $\boldsymbol{n} \perp \boldsymbol{B}$. The numbers denote the values of the absorption degree $q$. Descending polarization curves for $q=0.8$ and 0.9 are depicted as the dotted curves.

perpendicular to the plane $(\boldsymbol{n} \boldsymbol{N})$ and the Stokes parameter $Q^{(1 / 2)}<0$. At $q \approx 0.158$, when the angular distribution is practically isotropic, the polarization does not arise at all, $p_{\max }^{(1 / 2)}(\mu) \approx 0$. For $q>0.158$, the angular distribution looks as directed parallel to the surface and creates a "negative" polarization with the electric field oscillations in the scattering plane $(\boldsymbol{n} \boldsymbol{N})$. Such polarization by multiple scattering was first discovered by Nagirner (1962) and explained by Gnedin \& Silant'ev (1978). Up to $q \approx 0.5$, this negative polarization grows by $\left(p_{\max }^{(1 / 2)}(\mu=0, q=0.5)=0.52 \%\right)$ and then tends monotonically to zero for $q \longrightarrow 1$, when the scattering of photons disappears. It is of interest to note that numerical calculations of Bastien \& Ménard $(1988,1990)$ have also demonstrated the existence of negative polarization for the optically thick accretion disks consisting of Mie dust spheres.

Now consider Fig. 5, corresponding to a very thin source layer with $h=5$. For this case the angular distribution is "flat", mostly parallel to atmosphere's surface, and the polarization is negative for all values of $q$. Here the limiting case $q=0$ does not correspond to maximum polarization, as in the case of $h=0.5$. We have $p_{\max }^{(5)}(\mu=0, q=0)=1.853 \%$. Up to $q=0.05$ the polarization grows slightly $\left(p_{\max }^{(5)}(\mu=0, q=0.05)=2.259 \%\right)$ and then decreases up to zero for $q \longrightarrow 1$.

In Fig. 6 we give the curve dividing the plane of parameters $q$ and $h$ into regions with $Q^{(h)}(\mu)<0$, corresponding to the usual polarization with the electric oscillations perpendicular to the plane $(\boldsymbol{n} \boldsymbol{N})$, and the vast region of "negative" polarization. The curve itself corresponds to 


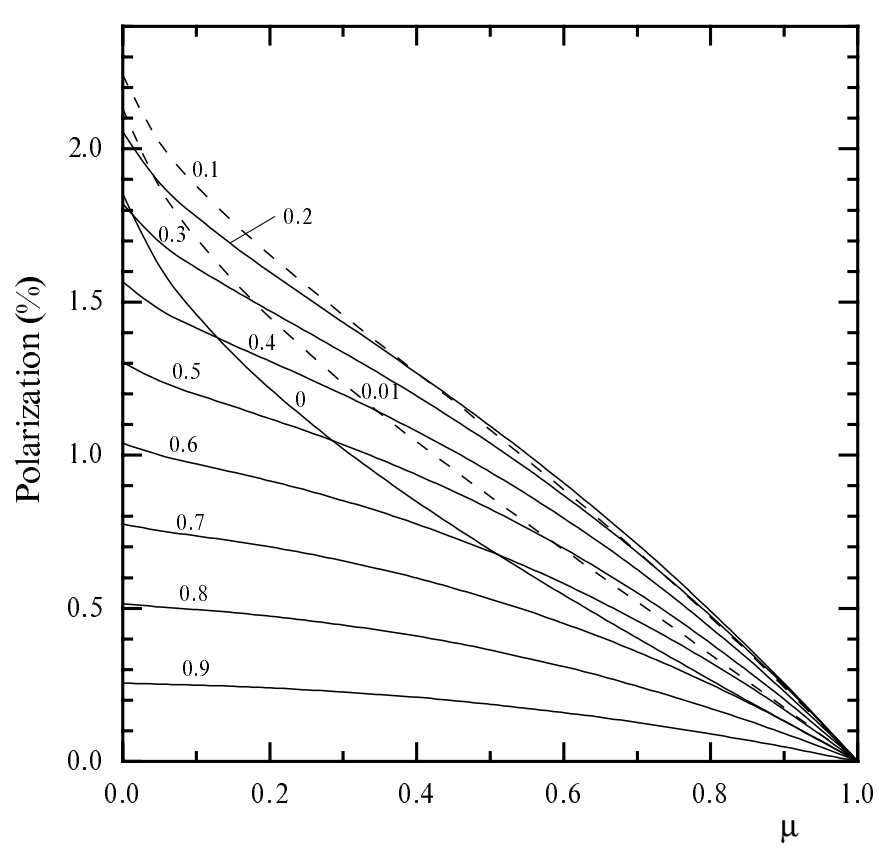

Fig. 5. Polarization for an atmosphere with exponential sources $(h=5)$ and for $\boldsymbol{n} \perp \boldsymbol{B}$. The numbers denote the values of the absorption degree $q$. The curves with $q=0.01$ and 0.1 for clarity are depicted as the dashed curves.

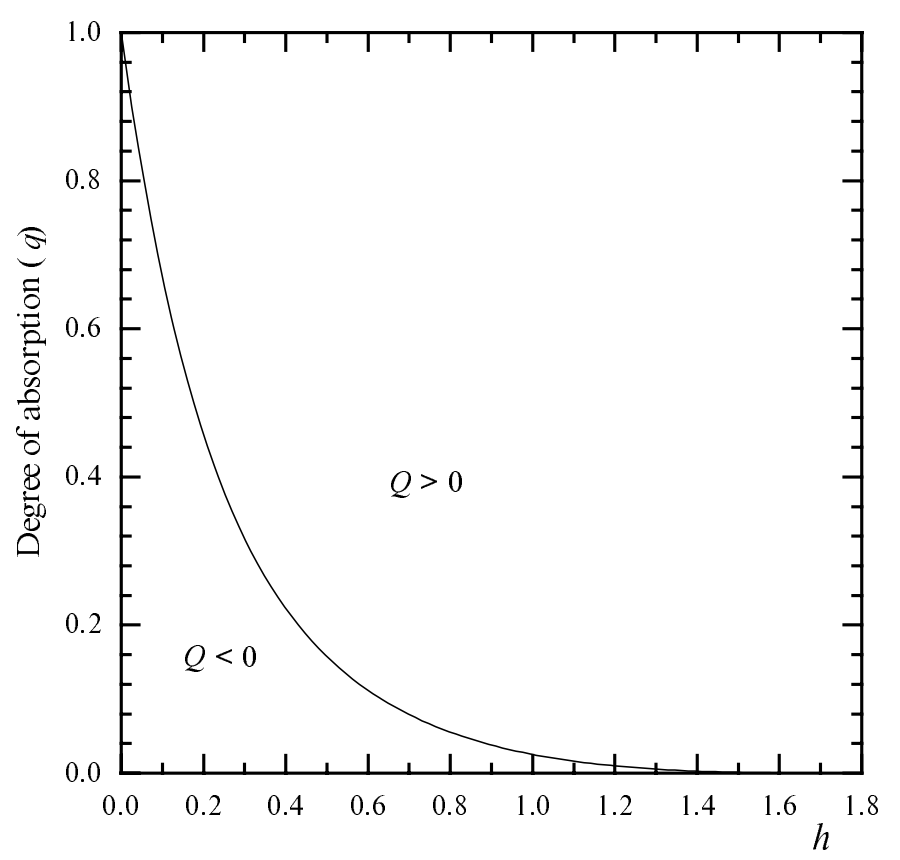

Fig. 6. The boundary between negative $(Q>0)$ and positive $(Q<0)$ polarization for atmospheres with exponential sources of the type $S(\tau)=q S_{h} \exp (-h \tau)$.

the absence of any polarization. For $h>1.65$ polarization is always negative. The absence of polarization for $h=0.5$ and 1 occurs at $q=0.158$ and $q=0.0254$, respectively. We note that our approximation $(1-q) \delta \geq 1$ gives rise to a change of sign of the Stokes parameter $Q^{(h)}(\mu, \cos \Theta=0)$ for all values of $\mu$. This is in contrast to the usual non-magnetized atmosphere, where for $\mu \approx 0$ the polarization is always "positive" (see Loskutov \& Sobolev 1981; Silant'ev 1980). Loskutov \& Sobolev (1981) give the polarization degree for the Milne problem and homogeneous and linearly distributed sources. Silant'ev (1980) also presents the case of exponential sources and the angular distributions for all considered atmospheres. Note that our calculations of angular distributions in a magnetized atmosphere are very close to those without any magnetic field. The degree of polarization $p_{\max }(\mu)$ qualitatively corresponds to the case of a non-magnetized atmosphere. The existence of the Faraday rotation gives rise to the peaklike form of polarization near $p_{\max }$, in accordance with the discussion in the Introduction.

\subsection{Approximation of the real distribution of sources}

If one has a model of the distribution of sources $S(\tau)$ of thermal radiation inside the accretion disk, we can approximate this distribution as a linear superposition of our "standard" sources.

$S(\tau) \approx q\left[S_{0}+S_{1} \tau+S_{h} \exp (-h \tau)\right]$

Due to the linearity of the radiation transfer equation, the intensity and the Stokes parameters, corresponding to the distribution (22), are the sum of standard solutions:

$I(\mu)=I^{(0)}(\mu)+I^{(1)}(\mu)+I^{(h)}(\mu)$,

$Q(\boldsymbol{n}, \boldsymbol{B})=Q^{(0)}(\boldsymbol{n}, \boldsymbol{B})+Q^{(1)}(\boldsymbol{n}, \boldsymbol{B})+Q^{(h)}(\boldsymbol{n}, \boldsymbol{B})$,

$U(\boldsymbol{n}, \boldsymbol{B})=U^{(0)}(\boldsymbol{n}, \boldsymbol{B})+U^{(1)}(\boldsymbol{n}, \boldsymbol{B})+U^{(h)}(\boldsymbol{n}, \boldsymbol{B})$.

Of course, we can take in (22)-(25) the sum over various values of the parameter $h$. Sometimes direct radiation from a central object is observed. In this case we add the corresponding intensity and Stokes's parameters to formulae $(23)-(25)$.

\subsection{Observed values}

To obtain the fluxes of radiation $F_{I}, F_{Q}$ and $F_{U}$ (erg $\mathrm{cm}^{-2} \mathrm{~s}^{-1} \mathrm{~Hz}^{-1}$ ), observed in a telescope, we integrate the values $I(\mu), Q(\boldsymbol{n}, \boldsymbol{B})$ and $U(\boldsymbol{n}, \boldsymbol{B})$ over all the observed surface of an accretion disk. In the general case all values $-\boldsymbol{B}, F, q, I, Q$ and $U$ depend on their position on a surface. Usually one supposes that the disk is flat, i.e. the outward normal $\boldsymbol{N}$ to the surface is independent of the position. In this case we can directly integrate parameters $Q(\boldsymbol{n}, \boldsymbol{B})$ and $U(\boldsymbol{n}, \boldsymbol{B})$, which refer to the system with its $x$-axis in the plane $(\boldsymbol{n} \boldsymbol{N})$. If an accretion disk is not flat, the $(\boldsymbol{n} \boldsymbol{N})$ - plane depends on its position on the curved surface. In this case we take into account the transformation law of the parameters $Q$ and $U$. Thus, what we observe with the telescope is:

$F_{I}=\frac{1}{R^{2}} \int \mathrm{d} A \mu I(\mu)$

$F_{Q}=\frac{1}{R^{2}} \int \mathrm{d} A \mu\left[Q(\boldsymbol{n}, \boldsymbol{B}) \cos 2 \varphi_{N}-U(\boldsymbol{n}, \boldsymbol{B}) \sin 2 \varphi_{N}\right]$ 
$F_{U}=\frac{1}{R^{2}} \int \mathrm{d} A \mu\left[U(\boldsymbol{n}, \boldsymbol{B}) \cos 2 \varphi_{N}+Q(\boldsymbol{n}, \boldsymbol{B}) \sin 2 \varphi_{N}\right]$.

Here, $R$ is the distance to the observer, $\mu=\cos \vartheta, \vartheta$ is the angle between the direction $\boldsymbol{n}$ to the observer and the normal $\boldsymbol{N}$ to the surface element $\mathrm{d} A, \varphi_{N}$ is the azimuthal angle of $\boldsymbol{N}$ in the telescope system (for a spherical surface $\boldsymbol{N}$ is parallel to the radius-vector $\boldsymbol{r}$ of the element $\mathrm{d} A=r^{2} \mathrm{~d} \vartheta \sin \vartheta \mathrm{d} \varphi$, so, $\left.\varphi_{N}=\varphi\right)$. The position dependence of the magnetic field $\boldsymbol{B}(\boldsymbol{r}, t)$ is the most important. Below we consider some particular cases of the magnetic field. Our simple analytical formulae for $Q$ and $U$ are not difficult to calculate numerically the $F_{Q}$ and $F_{U}$-values. Sometimes, if the azimuthal dependence of $\cos \Theta$ is simple, we can perform in (27)-(28) the integration over the azimuthal angle $\varphi$ analytically (see next section). Note that the general expression for $\delta \cos \Theta$ is:

$$
\begin{aligned}
\delta \cos \Theta & \simeq 0.8 \lambda^{2}(\mu \mathrm{m})\left[B_{z}(\rho, \varphi) \cos \vartheta\right. \\
& \left.+B_{\rho}(\rho, \varphi) \sin \vartheta \cos \varphi-B_{\varphi}(\rho, \varphi) \sin \vartheta \sin \varphi\right]
\end{aligned}
$$

Here, $\rho$ and $\varphi$ are cylindric coordinates of the surface element $\mathrm{d} A=\rho \mathrm{d} \rho \mathrm{d} \varphi$ at the flat disk (the $x, y$-plane).

\section{Simple models of magnetic fields in disks}

Consider the light polarization for some model distributions of magnetic fields in flat accretion disks. These models may be used for crude estimates by analysis of the observed polarization.

\subsection{Chaotic magnetic field}

If an accretion plasma is turbulent and the mean magnetic field is much smaller than the chaotic part of the field, we can average our formulae, considering that all directions of the magnetic field are equally probable. As a result of such averaging, the parameter $U(\boldsymbol{n}, \boldsymbol{B})$ disappears because direct and inverse magnetic fields are equally present in an atmosphere. In this situation the plane $(\boldsymbol{n N})$ is physically distinguished and, hence, the parameter $Q(\boldsymbol{n}, \boldsymbol{B}) \neq 0$. After averaging, we have:

$$
\begin{gathered}
Q^{(\mathrm{M})}(\boldsymbol{n}, B)=-\frac{F}{2 \pi J_{1}} \cdot \frac{1-g}{1+g} \cdot \frac{\left(1-\mu^{2}\right)}{(1-q) \delta} \arctan \frac{(1-q) \delta}{1-k \mu} \\
Q^{(0)}(\boldsymbol{n}, B)=-q S_{0} \frac{\left(1-\mu^{2}\right)\left(\alpha_{0}-\beta_{0}\right)}{2(1-q) \delta} \arctan (1-q) \delta \\
Q^{(1)}(\boldsymbol{n}, B)=-q S_{1} \frac{1-\mu^{2}}{2}\left[\frac{\mu\left(\alpha_{0}-\beta_{0}\right)}{1+(1-q)^{2} \delta^{2}}\right. \\
\left.+\frac{\alpha_{1}-\beta_{1}}{(1-q) \delta} \arctan (1-q) \delta\right] \\
Q^{(h)}(\boldsymbol{n}, B)=-q S_{h} \frac{\left(1-\mu^{2}\right)\left(\alpha_{h}-\beta_{h}\right)}{2(1-q) \delta} \arctan \frac{(1-q) \delta}{1+h \mu}
\end{gathered}
$$

Here, parameter $\delta$ depends on $B$ which is the mean value of a chaotic magnetic field. For $(1-q) \delta \gg 1$ all formulae acquire a simpler form, particularly, all values of $Q$ are proportional to $1 / \delta$. This does not mean that the polarization $\sim \lambda^{-2}$, because all other values $-q, \alpha_{n}$ and $\beta_{n}$, depend on $\lambda$. The value $B$ may also depend on the position on the surface of an accretion disk.

It should be noted that such an average has sense if the characteristic length of turbulence is greater than the free path of photons. In the opposite case of small-scale turbulence, the mean value of the Faraday rotation angle during the photon free path propagation is equal to zero, and the averaged inclination of the wave oscillation plane maintains its initial value. For small-scale turbulences only mean large-scale magnetic fields depolarize the linear polarization.

\subsection{Axially symmetric flat magnetic field}

If a magnetic field is axially symmetric and its force lines lie on the surface of an accretion disk $\left(B_{z}=0, B_{\rho}=B_{\rho}(\rho)\right.$ and $\left.B_{\varphi}=B_{\varphi}(\rho)\right)$, then

$\delta \cos \Theta=\delta \sin \vartheta \cos \left(\varphi+\varphi_{*}\right)$,

where $\cos \varphi_{*}=B_{\rho} /\left(B_{\rho}^{2}+B_{\varphi}^{2}\right)^{1 / 2}$ and $\delta$ depends on $B=$ $\left(B_{\rho}^{2}+B_{\varphi}^{2}\right)^{1 / 2}$ - the total magnitude of the flat magnetic field. This simple dependence on the azimuthal angle $\varphi$ allows us to carry out the $\varphi$-integration in (27) and (28) in an analytic form. Remember that for the flat disk we take $\mathrm{d} A=\rho \mathrm{d} \rho \mathrm{d} \varphi$ and $\varphi_{N}=0$. The axial symmetry of the magnetic field gives rise to zero values for all parameters $F_{U}$. For the remaining $F_{Q}$-parameters we obtain:

$$
\begin{aligned}
& F_{Q}^{(\mathrm{M})}=-\frac{\mu\left(1-\mu^{2}\right)}{R^{2}} . \\
& \int_{\rho_{1}}^{\rho_{2}} \mathrm{~d} \rho \rho \frac{(1-g) F(\rho)}{J_{1}(1+g) \sqrt{(1-k \mu)^{2}+(1-q)^{2} \delta^{2}\left(1-\mu^{2}\right)}} \\
& F_{Q}^{(0)}=-\frac{\pi \mu\left(1-\mu^{2}\right)}{R^{2}} . \\
& \int_{\rho_{1}}^{\rho_{2}} \mathrm{~d} \rho \rho \frac{q S_{0}\left(\alpha_{0}-\beta_{0}\right)}{\sqrt{1+(1-q)^{2} \delta^{2}\left(1-\mu^{2}\right)}}, \\
& F_{Q}^{(h)}=-\frac{\pi \mu\left(1-\mu^{2}\right)}{R^{2}} . \\
& \int_{\rho_{1}}^{\rho_{2}} \mathrm{~d} \rho \rho \frac{q S_{h}\left(\alpha_{h}-\beta_{h}\right)}{\sqrt{(1+h \mu)^{2}+(1-q)^{2} \delta^{2}\left(1-\mu^{2}\right)}}, \\
& F_{Q}^{(1)}=-\frac{\pi \mu\left(1-\mu^{2}\right)}{R^{2}} \text {. } \\
& \int_{\rho_{1}}^{\rho_{2}} \mathrm{~d} \rho \rho q S_{1}\left[\frac{\left(\alpha_{0}-\beta_{0}\right) \mu}{\left(1+(1-q)^{2} \delta^{2}\left(1-\mu^{2}\right)\right)^{3 / 2}}\right. \\
& \left.+\frac{\alpha_{1}-\beta_{1}}{\sqrt{1+(1-q)^{2} \delta^{2}\left(1-\mu^{2}\right)}}\right] \text {. }
\end{aligned}
$$




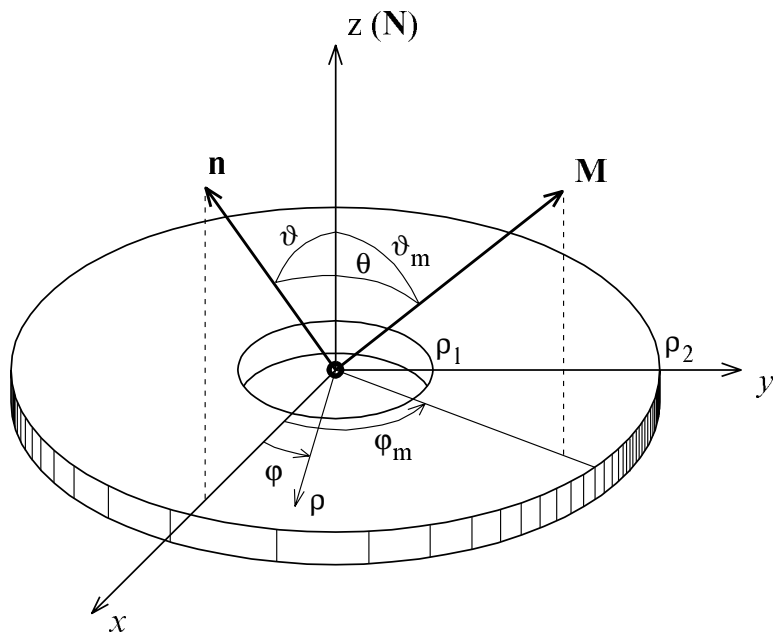

Fig. 7. The flat accretion disk (the $x, y$-plane) in the magnetic dipole field of a central source.

Here, $\rho_{1}$ and $\rho_{2}$ are inner and outer radii of the part of an accretion disk that give the main contribution to the energy flux for an observed range of wavelengths. We suppose that all parameters depend on the distance $\rho$, but do not depend on the azimuthal angle $\varphi$.

We see that all parameters $F_{Q}$ do not depend on the relative relation between radial and azimuthal components of the magnetic field. What is important is the radial dependence of the total magnetic energy which is described by the parameter $\delta^{2}$. Thus, for example, the radial magnetic field $\left(B_{\varphi}=0\right)$ and the azimuthal field $\left(B_{\rho}=0\right)$ give the same net polarization.

\subsection{Magnetic dipole field}

The interaction of the magnetic field of a central object with an accretion disk is a very complex problem (see, for example, Pringle \& Rees 1962; Lipunov \& Shakura 1980; Lai 1999; Bisnovatyi-Kogan 1999; Bisnovatyi-Kogan \& Lovelace 2000). Usually one assumes that the central object has a magnetic dipole field and studies the interaction of this field with the plasma of an accretion disk. For this reason, the model with a magnetic dipole field is of importance. Frequently one assumes that a magnetic dipole is perpendicular to the surface of a disk. In this case we have $\Theta=\vartheta$ on the surface and the azimuthal angle integration in (27)-(28) gives the factor $2 \pi$, for axially symmetric models. The Stokes parameters for oblique inclination of magnetic dipole are more difficult to calculate. Our simple analytic formulae for $Q(\boldsymbol{n}, \boldsymbol{B})$ and $U(\boldsymbol{n}, \boldsymbol{B})$ allow us to obtain the result of the azimuthal angle integration in an analytic form.

We consider the magnetic dipole $\boldsymbol{M}$ of a central object (neutron star, early type star etc.) characterized by the longitudinal and azimuthal angles $\vartheta_{m}$ and $\varphi_{m}$, respec- tively (see Fig. 7). As is well known, the magnetic field of the magnetic dipole has the form:

$\boldsymbol{B}(\boldsymbol{r}, t)=\frac{3(\boldsymbol{M} \cdot \boldsymbol{r}) \boldsymbol{r}-\boldsymbol{M} r^{2}}{r^{5}}$.

This expression gives the following magnetic field components on the surface of the flat disk:

$B_{z}=-B_{0} \cos \vartheta_{m}, \quad B_{\rho}=2 B_{0} \sin \vartheta_{m} \cos \left(\varphi_{m}-\varphi\right)$,

$B_{\varphi}=-B_{0} \sin \vartheta_{m} \sin \left(\varphi_{m}-\varphi\right), \quad B_{0}=M / \rho^{3}$.

According to the general expression (29), we have:

$(1-q) \delta \cos \Theta=\nu+\gamma \cos \left(2 \varphi-\varphi_{m}\right)$,

$\nu=(1-q) \delta\left[-\cos \vartheta \cos \vartheta_{m}+\frac{1}{2} \sin \vartheta \sin \vartheta_{m} \cos \varphi_{m}\right]$,

$\gamma=\frac{3}{2}(1-q) \delta \sin \vartheta \sin \vartheta_{m}$.

The parameter $\delta$ in (41) is equal to (1) with $B=B_{0}$. We also introduce the auxiliary values:

$\sigma_{M}=1-k \mu, \quad \sigma_{h}=1+h \mu, \quad \sigma_{0}=1, \quad \sigma_{1}=1$.

$2 b^{2}=\frac{\sigma^{2}+\nu^{2}-\gamma^{2}}{\sigma^{2}+(\nu-\gamma)^{2}}+\sqrt{\frac{\sigma^{2}+(\nu+\gamma)^{2}}{\sigma^{2}+(\nu-\gamma)^{2}}}$,

$c=a^{2}+b^{2}=\sqrt{\frac{\sigma^{2}+(\nu+\gamma)^{2}}{\sigma^{2}+(\nu-\gamma)^{2}}}$.

For every standard problem we use the corresponding $\sigma$ from (42).

After the azimuthal angle integration, formulae (27)(28) acquire the form:

$$
\begin{aligned}
& F_{Q}^{(\mathrm{M})}\left(\vartheta, \vartheta_{m}, \varphi_{m}\right)= \\
& -\frac{\mu\left(1-\mu^{2}\right)}{2 R^{2}} \int_{\rho_{1}}^{\rho_{2}} \mathrm{~d} \rho \rho \frac{(1-g) F \sigma_{M}(1+1 / c)}{(1+g) J_{1} b\left[\sigma_{M}^{2}+(\nu-\gamma)^{2}\right]} \\
& F_{U}^{(\mathrm{M})}\left(\vartheta, \vartheta_{m}, \varphi_{m}\right)= \\
& -\frac{\mu\left(1-\mu^{2}\right)}{2 R^{2}} \int_{\rho_{1}}^{\rho_{2}} \mathrm{~d} \rho \rho \frac{(1-g) F[\nu-\gamma+(\nu+\gamma) / c]}{(1+g) J_{1} b\left[\sigma_{M}^{2}+(\nu-\gamma)^{2}\right]} \\
& F_{Q}^{(0)}\left(\vartheta, \vartheta_{m}, \varphi_{m}\right)= \\
& -\frac{\pi \mu\left(1-\mu^{2}\right)}{2 R^{2}} \int_{\rho_{1}}^{\rho_{2}} \mathrm{~d} \rho \rho \frac{q S_{0}\left(\alpha_{0}-\beta_{0}\right)(1+1 / c)}{b\left[1+(\nu-\gamma)^{2}\right]} \\
& F_{U}^{(0)}\left(\vartheta, \vartheta_{m}, \varphi_{m}\right)=-\frac{\pi \mu\left(1-\mu^{2}\right)}{2 R^{2}} . \\
& \int_{\rho_{1}}^{\rho_{2}} \mathrm{~d} \rho \rho \frac{q S_{0}\left(\alpha_{0}-\beta_{0}\right)[\nu-\gamma+(\nu+\gamma) / c]}{b\left[1+(\nu-\gamma)^{2}\right]}
\end{aligned}
$$




$$
\begin{gathered}
F_{Q}^{(h)}\left(\vartheta, \vartheta_{m}, \varphi_{m}\right)=-\frac{\pi \mu\left(1-\mu^{2}\right)}{2 R^{2}} . \\
\int_{\rho_{1}}^{\rho_{2}} \mathrm{~d} \rho \rho \frac{q S_{h}\left(\alpha_{h}-\beta_{h}\right) \sigma_{h}(1+1 / c)}{b\left[\sigma_{h}^{2}+(\nu-\gamma)^{2}\right]} \\
F_{U}^{(h)}\left(\vartheta, \vartheta_{m}, \varphi_{m}\right)=-\frac{\pi \mu\left(1-\mu^{2}\right)}{2 R^{2}} . \\
\int_{\rho_{1}}^{\rho_{2}} \mathrm{~d} \rho \rho \frac{q S_{h}\left(\alpha_{h}-\beta_{h}\right)[\nu-\gamma+(\nu+\gamma) / c]}{b\left[\sigma_{h}^{2}+(\nu-\gamma)^{2}\right]} .
\end{gathered}
$$

The expressions $F_{Q}^{(1)}$ and $F_{U}^{(1)}$ have the most complex form:

$$
\begin{gathered}
F_{Q}^{(1)}\left(\vartheta, \vartheta_{m}, \varphi_{m}\right)=-\frac{\pi \mu\left(1-\mu^{2}\right)}{2 R^{2}} \\
\int_{\rho_{1}}^{\rho_{2}} \mathrm{~d} \rho \rho q S_{1}\left\{\frac{\alpha_{1}-\beta_{1}-\left(\alpha_{0}-\beta_{0}\right) \mu}{b\left[1+(\nu-\gamma)^{2}\right]}(1+1 / c)\right. \\
\left.\quad+\frac{8 \mu\left(\alpha_{0}-\beta_{0}\right)}{b\left[1+(\nu-\gamma)^{2}\right]^{2}}\left[D_{0}+C_{0}+\left(D_{1}+C_{1}\right) / 2 b^{2}\right]\right\} \\
F_{U}^{(1)}\left(\vartheta, \vartheta_{m}, \varphi_{m}\right)=-\frac{\pi \mu\left(1-\mu^{2}\right)}{2 R^{2}} \\
\int_{\rho_{1}}^{\rho_{2}} \mathrm{~d} \rho \rho q S_{1}\left\{\frac{\left(\alpha_{1}-\beta_{1}\right)[\nu-\gamma+(\nu+\gamma) / c]}{b\left[1+(\nu-\gamma)^{2}\right]}\right. \\
+\frac{8 \mu\left(\alpha_{0}-\beta_{0}\right)}{b\left[1+(\nu-\gamma)^{2}\right]^{2}}\left[\nu\left(D_{0}+C_{0}\right)+\gamma\left(D_{2}+C_{2}\right)\right. \\
\left.\left.+\frac{\nu}{2 b^{2}}\left(D_{1}+C_{1}\right) / 2 b^{2}+\gamma\left(D_{3}+C_{3}\right)\right]\right\}
\end{gathered}
$$

Here, functions $D_{n}$, and $C_{n}$ are:

$$
\begin{aligned}
& D_{0}=\frac{4 a^{2}\left(1+c^{3}\right)+c(1-c)^{3}}{16 a^{2} c^{3}}, \\
& C_{0}=\frac{4 a^{2}\left(c^{3}-1\right)+c(c-1)^{3}}{32 a^{2} c^{3}}, \\
& D_{1}=\frac{4 a^{2}\left(1-c^{3}\right)+c(c-1)^{3}}{16 a^{2} c^{2}}, \\
& C_{1}=\frac{4 a^{2} c^{2}-2 c^{3}+3 c^{2}-1}{8 c^{2}}, \\
& D_{2}=\frac{4 a^{2}\left(1-c^{3}\right)+c-c^{2}-c^{3}+c^{4}}{16 a^{2} c^{3}} \\
& C_{2}=\frac{-4 a^{2}\left(1+c^{3}\right)-c+c^{2}+c^{3}-c^{4}}{32 a^{2} c^{3}} \\
& D_{3}=\frac{4 a^{2}\left(1+c^{3}\right)-c+c^{2}+c^{3}-c^{4}}{16 a^{2} c^{2}}, \\
& C_{3}=\frac{2 c^{3}-c^{2}-1-4 a^{2} c^{2}}{8 c^{2}} .
\end{aligned}
$$

As we have mentioned, for $\vartheta_{m}=0$ one has $\Theta=\vartheta$, and azimuthal integration gives the factor $2 \pi$. It is easy obtain this result from our general complex formulae. In this case we have $\gamma=0, b=1, c=1$ and $a=0$. Formally, most coefficients in (53) diverge. But in formulae (51) and (52) these diverging coefficients cancel each other and we obtain the needed $2 \pi$-coefficient. For crude estimates one can neglect the $\rho$-dependence of all values. Then the $\rho$ integration in (45)-(52) gives the value $A / 2 \pi$ with $A$ being the area of a disk.

\subsection{Diamagnetic disk}

For diamagnetic (perfectly conducting) plasma the normal $z$-component of magnetic field does not penetrate inside the disk (see Lai 1999). Practically this occurs if the time of molecular diffusion, depending on a conductivity, is much shorter than other dynamical characteristic times (period of cyclotron rotation, time of turbulent mixing etc.). For this case we can omit the factor $\left(-\cos \vartheta \cos \vartheta_{m}\right)$ in the $\nu$-coefficient in (41). Considering that the most important dependence on $\rho$ occurs due to the factor $B_{0}=M / \rho^{3}$ in $\delta$, and taking out the integrands all other quantities in the mean value of $\rho$, we obtain the following formula for the degree of polarization:

$$
\begin{aligned}
p\left(\vartheta, \vartheta_{m}, \varphi_{m}\right)=\frac{4}{15} \cdot \frac{\alpha-\beta}{\alpha+\beta} . \\
\frac{\sqrt{1-\mu^{2}}}{J(\mu)(1-q) \delta_{2} \sin \vartheta_{m} \sqrt{1-\left(\cos ^{2} \varphi_{m}\right) / 9}}
\end{aligned}
$$

Here $\delta_{2}$ is parameter (1) taken for a magnetic field at $\rho=\rho_{2}$ - the outer radius of the accretion disk. We neglect the contribution of the inner radius $\rho_{1}<\rho_{2}$, considering $\rho_{2}^{5} \gg \rho_{1}^{5}$. This formula applies to all "standard" problems, i.e. we have to take $\alpha=\alpha_{0}, \alpha_{1}, \alpha_{h}$ and analogously for the $\beta$-coefficients. For the Milne problem one must take $\alpha=1$ and $\beta=g$. Note that $J(\mu) \equiv I(\mu) / I(0)$.

The simple formula (54) was derived under the assumption that the parameter

$$
\frac{5}{33(1-q)^{2} \delta_{2}^{2}\left(1-\mu^{2}\right) \sin ^{2} \vartheta_{m}} \ll 1 \text {. }
$$

We see that the dependence of polarization on the azimuthal angle $\varphi_{m}$ is weak. We note also that in this approximation all $U$-parameters are equal to zero.

It is interesting that for very large Faraday's rotation $((1-q) \delta \gg 1)$ the polarization degree is proportional to $1 /(1-q) \delta$ for all problems. This dependence has a simple explanation. In a non-magnetized atmosphere the outgoing radiation is mostly collected from the total optical depth $\tau \approx 1$. For the intensity $I$ this is true also for an atmosphere with Faraday rotation. For large Faraday's rotation the values of the Stokes parameters $Q$ and $U$ are determined by the contribution of a thin surface layer, where the angle of rotation $\psi \leq 1$ (see Eq. (1)). The optical depth of this layer $\tau_{\text {pol }} \approx 1 /(1-q) \delta \ll 1$. Thus, the $Q$ and $U$-parameters are proportional to $\tau_{\text {pol }} \sim 1 /(1-q) \delta$. If $\tau_{\text {pol }}>1$, the Faraday rotation does not affect the polarization of the outgoing radiation. 


\section{Conclusion}

Using the assumption of a large Faraday rotation (the Faraday rotation angle $\psi \geq 1$ at the optical length $\tau \approx 1$ ), we obtained simple approximate formulae for the intensity and the Stokes parameters of outgoing radiation for a number of "standard" problems (the Milne problem, the atmospheres with homogeneous and linear distribution of thermal sources as well as exponentially decreasing distribution of sources in a magnetized, plane-parallel, optically thick atmosphere). The polarization arises as a result of the light scattering on free electrons in a magnetized plasma of an atmosphere. The magnetic field is assumed to be $<10^{5} \mathrm{G}$, when the scattering cross-sections are equal to the Thomson value. Practically any real distribution of sources in such atmospheres can be approximated as a superposition of these standard sources. Thus, our formulae allow us to obtain the polarization for various real atmospheres. Of course, the distribution of sources in disks depends also on the illumination from the central objects (star, quasar etc.). Sometimes, direct radiation from these central objects must be also taken into account and the corresponding intensity and the Stokes parameters are to be added to our formulae. The most suitable objects to apply our simple formulae are hot accretion disks around quasars and active galactic nuclei (see Wills et al. 1992 and references therein). The characteristic spectra of polarization, corresponding to atmospheres with Faraday rotation, can be used to confirm the existence of magnetic fields in these disks and to give some estimates of its values.

It was shown that the Faraday rotation very effectively depolarizes the radiation inside the optically thick atmosphere and to obtain the polarization of outgoing radiation, one can consider only the last scattering of nonpolarized radiation before the escape from an atmosphere. In this approximation the intensity of radiation satisfies the separate transfer equation with Rayleigh's phase function.

The classic methods of Chandrasekhar and Sobolev allowed us to express all values as depending on one scalar $H$-function. This function can be easily calculated from the known Ambartzumian - Chandrasekhar nonlinear equation. We numerically calculated all required values and present the results in tables and figures.

We also present the calculation of polarization for some simple models of the magnetic field distribution in an accretion disk (chaotic field in turbulent plasma, axially symmetric plane magnetic field and the field of magnetic dipole). For a diamagnetic (perfectly conducting) accretion disk in the dipole field of a central object we obtained very simple asymptotic formulae for polarization, depending on the longitudinal and azimuthal angles of the magnetic dipole position and the angle of observation.
These results allow us to calculate and estimate the polarization for a large variety of models of optically thick, magnetized accretion disks.

\section{References}

Agol, E., \& Blaes, O. 1996, MNRAS, 282, 965

Agol, E., Blaes, O., \& Ionescu-Zanetti, C. 1998, MNRAS, 293, 1

Basko, M. A. 1977, Afz, 12, 169

Bastien, P., \& Ménard, F. 1988, ApJ, 326, 334

Bastien, P., \& Ménard, F. 1990, ApJ, 364, 232

Bisnovatyi-Kogan, G. S. 1999, Odessa Astron. Publ., 12, 169

Bisnovatyi-Kogan, G. S., \& Lovelace, R. V. E. 2000, ApJ, 529, 978

Chandrasekhar, S. 1950, Radiative Transfer (Clarendon Press, Oxford), 89

Frank, J., King, A., \& Raine, D. 1992, Accretion power in astrophysics (Cambridge, Cambridge Univ. Press)

Gnedin, Yu. N., \& Silant'ev, N. A. 1997, Basic mechanisms of light polarization in cosmic media (Hartwood Academic Publ., Amsterdam), 30

Gnedin, Yu. N., \& Silant'ev, N. A. 1978, AZh, 55, 564

Hutsemékers, D., Lamy, H., \& Remy, M. 1998, A\&A, 340, 371

Kaminker, A. D., Pavlov, G. G., \& Shibanov, Yu. A. 1982, Ap\&SS, 86, 249

Korotkar, A., Antonucci, R. R., Goodrich, R. W., Bushouse, H., \& Kinney, A. L. 1995, ApJ, 450, 501

Lai, D. 1999, ApJ, 524, 1030

Lipunov, V. M., \& Shakura, N. M. 1980, Sov. Astron. Lett., 6, 14

Loskutov, V. M., \& Sobolev, V. V. 1981, Afz, 17, 97

Meszaros, P., Nagel, W., \& Ventura, J. 1980, ApJ, 238, 1066

Moore, R. L., \& Stockman, H. S. 1984, ApJ, 279, 465

Nagel, W. 1980, ApJ, 236, 904

Nagirner, D. 1962, Trudi Astron. Observ. Leningrad University, 30, 79

Pringle, J. F., \& Rees, M. J. 1972, A\&A, 21, 1

Schmidt, G. D., Hines, D. C., \& Smith, P. S. 1997, in Mass ejection from active galactic nuclei, ed. N. Arav, I. Shlosman, \& R. J. Waymann, ASP Conf. Ser., 128, 106

Silant'ev, N. A. 1979, SvA, 23, 21

Silant'ev, N. A. 1980, AZh, 57, 587

Silant'ev, N. A. 1982, Ap\&SS, 82, 363

Silant'ev, N. A. 1994, JQSRT, 52, 207

Sobolev, V. V. 1960, SvA, 3, 563

Sobolev, V. V. 1972, The light scattering in the atmospheres of planets, in russian (Nauka, Moscow), 355

Stockman, H. S., Moore, R. L., \& Angel, J. R. P. 1984, ApJ, 279,485

Vallée, J. P. 1997, Fund. Cosm. Phys., 19, 1

Webb, W., Malkan, M., Schmidt, G., \& Impey, C. 1993, ApJ, 419, 494

Wills, B. J., Wills, D., Breger, M., Antonucci, R. R. J., Barvians, R. 1992, ApJ, 398, 454

Yudin, R. V. 1996, A\&A, 312, 234 